\title{
GRADIENTS OF ELECTRON TEMPERATURE AND DENSITY ACROSS $m=2$ MAGNETIC ISLANDS IN RTP
}

\author{
B.PH. VAN MILLIGEN, A.C.A.P. VAN LAMMEREN, N.J. LOPES CARDOZO, \\ F.C. SCHÜLLER, M. VERRECK \\ FOM Institute for Plasma Physics "Rijnhuizen", \\ Nieuwegein, \\ The Netherlands
}

\begin{abstract}
Simultaneous measurements of oscillations of the electron temperature, the electron density and the poloidal magnetic field in the Rijnhuizen Tokamak RTP are combined to obtain insight into the structure of MHD mode perturbations. Diagnostics used are a 20 channel heterodyne ECE radiometer, a 19 channel FIR interferometer, and a set of magnetic pick-up coils. $\mathrm{A} \mathrm{m} / \mathrm{n}=2 / 1$ island preceding a disruption is analysed in detail. Whereas the density and temperature are significantly lower in the O-point than in the X-point, no local minimum or even flattening of the profiles could be demonstrated. The gradients across the island may be understood in terms of a very long connection length inside the island, in combination with the relatively short mean free path along the field lines due to the high collisionality in the outer region of the plasma. The transport inside the island and the poloidal distribution of the radial heat flux over X-and O-point are discussed.
\end{abstract}

\section{INTRODUCTION}

In this paper observations of magnetic islands are reported. The observations are done on the Rijnhuizen Tokamak RTP, which is dedicated to the study of transport, and is equipped with diagnostics for detailed measurements of the electron temperature $\left(\mathrm{T}_{\mathrm{e}}\right)$ and density $\left(\mathrm{n}_{\mathrm{e}}\right)$.

Magnetohydrodynamis (MHD) theory describes magnetic islands as helical systems of closed magnetic surfaces. The expectation is that if such islands occur, the electron temperature and pressure $\left(\mathrm{p}_{\mathrm{e}}\right)$ are constant on the flux surfaces that form the island. Thus the island would show up as a region where the $\mathrm{T}_{\mathrm{e}}$ and $\mathrm{p}_{\mathrm{e}}$ profiles show symmetry around the magnetic axis of the island. With sufficiently detailed measurements, it could be possible to determine the temperature and pressure profiles inside the island. Detailed experimental observations of large magnetic islands mainly concern the $(m, n)=(1,1)$ pre- and post cursors of the sawtooth instability [1], the $\mathrm{m} / \mathrm{n}=1$ region of high density observed in JET (the so-called snake) [2], and the $(\mathrm{m}, \mathrm{n})=(2,1)$ mode that occurs as a precursor of a major disruption [3-6]. In the case of the $(\mathrm{m}, \mathrm{n})=(1,1)$ islands, measurements of $\mathrm{T}_{\mathrm{e}}$ confirmed the theoretical expectation [1], with $\mathrm{T}_{\mathrm{e}}$-contours mapping out the island structure. In the case of the $(m, n)=(2,1)$ mode, however, the situation is more ambiguous. Flat spots in the $\mathrm{T}_{\mathrm{e}}$ profile are consistently observed at high $\mathrm{T}_{\mathrm{e}}\left(\mathrm{T}_{\mathrm{e}} \geq 500 \mathrm{eV}\right)$ on JET [3, 5, 7] and TFTR [8]. In [9] observations of flat spots in the density profile in TEXT are reported. At low $\mathrm{T}_{\mathrm{e}}\left(\mathrm{T}_{\mathrm{e}} \leq 400 \mathrm{eV}\right)$, however, incomplete flattening is seen on JET [4], TEXTOR [6] and Wendelstein VII-A [10]. More specifically, small shoulders in the $\mathrm{T}_{\mathrm{e}}$ profile are reported on TEXTOR [6] that appear when the $\mathrm{m}=2$ mode locks to the wall and disappear when the mode unlocks. Although no magnetic measurements are presented in [6], it is likely that the island in those conditions is substantially wider than the observed flat area in the $T_{e}$ profile. On Wendelstein VII-A, the island width as deduced from magnetic measurements exceeds the island width as found from $\mathrm{T}_{\mathrm{e}}$ and $\mathrm{n}_{\mathrm{e}}$ profile measurements substantially, although the difference decreases with decreasing mode rotation velocity [10]. In [5] an analysis is given of the fluxes inside an island, which resulted in an estimation of the minimal size of an island that would lead to flat spots in the $\mathrm{T}_{\mathrm{e}}$ profile in typical JET disruption precursors.

The present paper investigates the possibility of sustained $\mathrm{T}_{\mathrm{e}}$ and $\mathrm{p}_{\mathrm{e}}$ gradients across $\mathrm{m}=2$ islands in more detail. Measurements of both $\mathrm{T}_{\mathrm{e}}$ and $\mathrm{n}_{\mathrm{e}}$ in a very large $(m, n)=(2,1)$ island preceding a disruption are presented and analysed. The location of the $\mathrm{T}_{\mathrm{e}}$ and $\mathrm{n}_{\mathrm{e}}$ perturbations are compared to the position and width of the island as deduced from magnetic measurements, and we investigate whether there are closed isothermal and isobaric surfaces within the island. Further, the incremental current in the island O-point is estimated, and the coupling between the $m=1$ and $m=2$ modes is considered. A model for the heat flow inside a magnetic island is presented, which accounts for the fluxes along and across the field lines. This treatment has a resemblance to the analysis in [5], although both the method of analysis and - more importantly - the results are rather different.

In Section 2, the diagnostics and some aspects of the data analysis methods are described. The experimental results are presented in Section 3, the 
model for the heat flow through the island is presented in Section 4. The results are discussed in Section 5.

\section{DIAGNOSTICS AND DATA ANALYSIS METHODS}

\subsection{The RTP tokamak and diagnostics}

A schematic top view of RTP is shown in Fig. 1. The major radius is $0.72 \mathrm{~m}$, the minor radius of the vessel $0.24 \mathrm{~m}$ and the plasma horizontal minor radius is $0.165 \mathrm{~m}$. The position of the circular poloidal limiter and the moveable top-bottom limiters are indicated. Seen from the top, both the plasma current $\left(\mathrm{I}_{\mathrm{p}}\right)$ and the toroidal magnetic field $\left(\mathrm{B}_{\phi}\right)$ are directed clockwise. In ohmic discharges, typical plasma parameters are: $\mathrm{I}_{\mathrm{p}} \leq$ $150 \mathrm{kA}, \mathrm{B}_{\phi} \leq 2.4 \mathrm{~T}, \mathrm{n}_{\mathrm{e}}(0) \leq 1 \cdot 10^{20} \mathrm{~m}^{-3}, \mathrm{~T}_{\mathrm{e}}(0)=0.8$ $\mathrm{keV}, \tau_{\mathrm{E}} \leq 6 \mathrm{~ms}$. The pulse length is typically $300 \mathrm{~ms}$.

Diagnostics available at RTP include: magnetics, neutral particle analyser, soft X-ray pulse height analysis and tomography, ECE heterodyne radiometer (20 channels) and grating polychromator (6 channels), $2 \mathrm{~mm}$ interferometer, 19-channel FIR interferometer. RTP is equipped with a multiple pellet injector and ECRH (2 gyrotrons, $60 \mathrm{GHz}, 200 \mathrm{~kW}$ each, $100 \mathrm{~ms}$ pulse length, O-mode low-field-side launch and X-mode high-field-side launch, respectively).

The magnetic diagnostic consists of a set of 12 poloidal field pick-up coils and 12 saddle or flux loops. The poloidal field pick-up coils are mounted in a ring with a minor radius of $0.213 \mathrm{~m}$. The transfer function of the pick-up coils has its $3 \mathrm{~dB}$ point at 10 $\mathrm{kHz}$.

The 20-channel radiometer [11] measures $2^{\text {nd }}$ harmonic ECE X-mode, from which $\mathrm{T}_{\mathrm{e}}$ is deduced. The antenna is located at $\mathrm{R}=0.955 \mathrm{~m}$. The radial resolution is $\approx 2 \mathrm{~cm}$ at $\mathrm{R}=0.72 \mathrm{~m}, 1.5 \mathrm{~cm}$ at $\mathrm{R}=$ $0.62 \mathrm{~m}$, and $2.5 \mathrm{~cm}$ at $\mathrm{R}=0.82 \mathrm{~m}$. The perpendicular resolution (full $3 \mathrm{~dB}$ beam width) is $5.2^{\circ}$ in the poloidal direction and $7^{\circ}$ in the toroidal direction, the minimal spot size is $2 \times 2 \mathrm{~cm}$. The positions of the channels, on average $2 \mathrm{~cm}$ apart, are computed from the value of the vacuum toroidal magnetic field. The paramagnetic and poloidal fields are neglected, which may lead to systematic errors of a few $\mathrm{mm}$ in the discharges under study. The noise equivalent temperature is typically $2-3 \mathrm{eV}$ at the video-bandwidth $(20 \mathrm{kHz})$ used in the discharges under study. The computation of $\mathrm{T}_{\mathrm{e}}$ includes optical depth effects, using the Abel-inverted $\mathrm{n}_{\mathrm{e}}$ profiles from the interferometer.

The 19 channels of the multi-channel interferometer [12] have variable spacing. For the discharge under study, the spacing of the 14 available channels was $2.4 \mathrm{~cm}$ on average. The FIR laser wavelength is $\lambda=432 \mu \mathrm{m}$, the intermediate frequency between the plasma and reference beams is $1 \mathrm{MHz}$, allowing high time resolution. The accuracy in the line-integrated density is better than $2 \cdot 10^{16} \mathrm{~m}^{-2}$.

\subsection{Data analysis methods}

Magnetic islands are associated with surfaces where the safety factor ( $q$ ) is rational: $q=m / n$. For simplicity, we consider an equilibrium with circular concentric nested flux surfaces in cylindrical approximation. We adopt the usual polar coordinate system $(r, \theta, \phi)$ (where $\phi$ is the toroidal coordinate) related to the cylindrical coordinate system $(R, Z, \phi)$ by $R=R_{0}+r \cos \theta$ and $Z$ $=r \sin \theta \cdot R_{0}$ is the major radius.

The radial island width (w), i.e. the distance between the separatrices at either side of the O-point, can be expressed in terms of the radial field perturbation at the rational surface [13]:

$$
\mathrm{w}=4\left(\frac{\mathrm{rq} \hat{\mathrm{B}}_{\mathrm{r}}}{\mathrm{m \textrm {q } ^ { \prime } \mathrm { B } _ { \theta }}}\right)^{\frac{1}{2}},
$$

where $\hat{B}_{r}$ is the amplitude of the radial field perturbation due to the island, $\mathrm{q}^{\prime}=\partial \mathrm{q} / \partial \mathrm{r}$, and all quantities are to be evaluated at the radius $r_{m ; n}$ of the rational q-surface.

Since there is no direct measurement of the local $\hat{B}_{r}\left(r=r_{m} ; n\right)$, this has to be deduced from measurements of either $\mathrm{B}_{\mathrm{r}}$ or $\mathrm{B}_{\theta}$ outside the plasma

$$
\begin{aligned}
& \hat{B}_{r}\left(r_{m ; n}\right)=\hat{B}_{r}\left(r_{\text {coils }}\right)\left(\frac{r_{\text {coils }}}{r_{m ; n}}\right)^{m+1} \\
& \hat{B}_{r}\left(r_{\text {coils }}\right)=\hat{B}_{\theta}\left(r_{\text {coils }}\right)
\end{aligned}
$$

where $r_{\text {coils }}$ denotes the minor radius of the ring of pick-up coils. The radius $r_{m ; n}$ of the rational q-surface can be deduced from the q-profile, which in the outer part of the plasma is known with sufficient accuracy.

The rotating helical structures give rise to a signal on the poloidal field pick-up coils given by

$$
\begin{aligned}
& \frac{\partial}{\partial t} \hat{B}_{\theta}(\theta, \phi, t)=\sum_{m}^{n} \hat{B}_{\theta m ; n} \omega_{m ; n} \times \\
& \cos \left(m \theta-n \phi-\chi_{m ; n}-\omega_{m ; n} t\right)
\end{aligned}
$$

To separate the contributions of different poloidal mode numbers to the observed oscillations, a Fourier analysis with respect to the poloidal angle $\theta$ of the pick-up coils is applied. We define

$$
\begin{aligned}
& \mathrm{F}_{\mathrm{m}}^{\mathrm{c}}=\frac{1}{\pi} \int_{0}^{2 \pi} \frac{\partial}{\partial \mathrm{t}} \hat{\mathrm{B}}_{\theta}(\theta, \phi, \mathrm{t}) \cos (\mathrm{m} \theta) \mathrm{d} \theta \\
& \mathrm{F}_{\mathrm{m}}^{\mathrm{s}}=\frac{1}{\pi} \int_{0}^{2 \pi} \frac{\partial}{\partial \mathrm{t}} \hat{\mathrm{B}}_{\theta}(\theta, \phi, \mathrm{t}) \sin (\mathrm{m} \theta) \mathrm{d} \theta
\end{aligned}
$$


In practice the integrals $(4 a, b)$ are replaced by sums over the pick-up coils. These Fourier coefficients are combined to give the combined amplitude $\left(\mathrm{S}_{\mathrm{m}}\right)$ of all modes of poloidal mode number $\mathrm{m}$.

$$
S_{m}=\sqrt{\left(F_{m}^{c}\right)^{2}+\left(F_{m}^{s}\right)^{2}}=\sum_{n} \hat{B}_{n}^{\theta m} \omega_{m ; n}
$$

The $\mathrm{S}_{\mathrm{m}}$ are used to monitor the evolution of the different m-modes (see Fig. 3). If we know that the dominant contribution to $\mathrm{S}_{\mathrm{m}}$ is due to the $\mathrm{n}=1$ mode, we may use $S_{m} / \omega_{m ; 1}$ as the experimental estimator for $\hat{B}_{n}^{\theta m}\left(r_{\text {coils }}\right)$.

To estimate the amount of current associated with the MHD-modes, we let the axisymmetric current density be perturbed by incremental current densities that are located on the rational q surfaces:

$$
\begin{aligned}
& \frac{\mathrm{dI}}{\mathrm{d} \theta}(\mathrm{r}, \theta, \phi)=\sum_{\mathrm{m}}^{\mathrm{n}} \frac{\mathrm{m}}{2} \delta \mathrm{I}_{\mathrm{m} ; \mathrm{n}} \times \\
& \sin \left(\mathrm{m} \theta-\mathrm{n} \phi-\chi_{\mathrm{m} ; \mathrm{n}}-\omega_{\mathrm{m} ; \mathrm{n}} \mathrm{t}\right) \delta\left(\mathrm{r}-\mathrm{r}_{\mathrm{m} ; \mathrm{n}}\right)(6)
\end{aligned}
$$

where $\delta \mathrm{I}_{\mathrm{m} ; \mathrm{n}}$ is the incremental current flowing in one of the $\mathrm{m} \mathrm{O}$-point regions of the island chain, $\chi_{\mathrm{m} ; \mathrm{n}}$ is the phase of island chains on separate flux surfaces and can be taken equal to zero if only one mode is described, $\omega_{\mathrm{m} ; \mathrm{n}}$ is the rotation velocity of the island. The $\delta$-function $\delta\left(r-r_{m ; n}\right)$ is an approximation of the radial current localization on the flux surface.

The amplitude $\hat{\mathrm{B}}^{\theta \mathrm{m}}$ of the poloidal field oscillation is related to the incremental current $\delta \mathrm{I}_{\mathrm{m} ; \mathrm{n}}$, in cylindrical approximation, by

$$
\delta I_{m ; n}=\hat{B}_{n}^{\theta m}\left(r_{\text {coils }}\right) 4 \frac{r_{m ; n}}{\mu_{0} m}\left(\frac{r_{\text {coils }}}{r_{m ; n}}\right)^{m+1}
$$

for $m \geq 1$.

If a single mode dominates the magnetic field oscillation or if simultaneous modes can be distinguished by means of their frequencies, eq. (7) allows the evaluation of the incremental current $\delta \mathrm{I}_{\mathrm{m} ; n}$. The direction of the incremental current depends on the sign of the shear $s=r q / q$. If $s>0$ at the surface with $q=$ $\mathrm{m} / \mathrm{n}$, the incremental current flowing in the O-point regions of an island chain is anti-parallel to the main current $\mathrm{I}_{\mathrm{p}}$, for $\mathrm{s}<0$ the incremental current in the $\mathrm{O}$ points is parallel to $I_{p}$.

\section{EXPERIMENTAL RESULTS}

We focus on one particular discharge of which the general characteristics are discussed in section 3.1.
Detailed analysis of the strong MHD activity that precedes a disruption is presented in section 3.2. Observations very similar to these, both in character and in magnitude, have been made in a number of other disrupting RTP discharges.

\subsection{Discharge R19910423.057}

Discharge R19910423.057 is a low-q ( $(\mathrm{a})=2.8)$ discharge without additional heating. Fig. 2 shows the plasma current, the central line integrated electron density, and the central $\mathrm{T}_{\mathrm{e}}$. A disruption occurs at $\mathrm{t}=$ $147 \mathrm{~ms}$. Sawteeth are observed on both $\mathrm{T}_{\mathrm{e}}$ and $\mathrm{n}_{\mathrm{e}}$ preceding the onset of strong MHD mode activity.

\subsection{Observations of MHD activity}

\subsubsection{Characterisation of the activity}

Fig. 3 shows the MHD activity signals computed according to eq. (6) for $m=1,2$ and 3 . It is seen that the MHD mode activity is dominantly $\mathrm{m}=2$ preceding the minor disruption at $\mathrm{t}=144 \mathrm{~ms}$. From analysis of the pick-up coils it is found, under the assumption that the mode structure rotation is dominantly toroidal, that the mode rotates in the electron drift direction. Fig. 4 shows the evolution of the dominant frequency of the signal of ECE channel 7 at $\mathrm{R}=0.819 \mathrm{~m}$. The mode slows down linearly in time prior to the disruption, in agreement with previous observations in JET [4].

\subsubsection{Location and width of the $m=2$ island, deduced from magnetics}

To compute $\mathrm{r}(\mathrm{q}=2)$ and $\mathrm{q}^{\prime}$ at that radius, we assume a parabolic q-profile, in agreement with profileconsistency considerations [14]. The edge value $\mathrm{q}=2.80$ at $\mathrm{a}=0.165 \mathrm{~m}$, and the sawtooth inversion radius are used to fix the coefficients. The $\mathrm{T}_{\mathrm{e}}$-sawteeth preceding the $\mathrm{m}=2$ mode activity show an inversion at $\mathrm{R}=0.67$ $\mathrm{m}$, corresponding to $\mathrm{r}=0.055 \mathrm{~m}$. Thus the q-profile is given by

$$
q(r)=0.78+2.01(r / a)^{2}
$$

Using this estimate of the $\mathrm{q}$-profile yields $\mathrm{r}(\mathrm{q}=2)=0.13$ $\mathrm{m}$.

The amplitude of the $\mathrm{m}=2$ mode $\mathrm{S}_{2}=47$ $\mathrm{Ts}^{-1}$ (Fig. 3) and from the frequency of the mode at $\mathrm{t}=$ $0.142 \mathrm{~s}$ of $f=4.7 \mathrm{kHz}$ it follows (eq. (5)) that $\hat{\mathrm{B}}_{\theta 2 ; 1}$ $=1.6 \times 10^{-3} \mathrm{~T}$. Using eq. (8) to compute $\mathrm{q}^{\prime}$ and $r_{\text {coils }}=0.213 \mathrm{~m}$, the island width and the incremental current can be evaluated using eqs. (1) and (7). Summarizing the results of the magnetic measurements:

$$
\begin{aligned}
& \mathrm{r}(\mathrm{q}=2)=0.13 \mathrm{~m} \\
& \mathrm{w}=6.4 \pm 0.5 \mathrm{~cm} \\
& \delta \mathrm{I}_{2 ; 1}=1.5 \mathrm{kA}
\end{aligned}
$$


No correction has been made for toroidal effects, which flatten the island on the low field side. Thus, w on the low field side is overestimated by $\approx 20 \%$. Also, no account has been taken of the influence of presence of the vessel wall on the poloidal field measurements. A perfectly conducting wall in our geometry would reduce $\mathrm{B}_{\theta 2 ; 1}$ for a stationary mode by about $50 \%$. This means that $\mathrm{w}$ would be underestimated by $30 \%$. For the rotating mode at $f=4.7 \mathrm{kHz}$ this correction will be reduced somewhat.

\subsubsection{Deformation of the $T_{e}$ and $n_{e}$ profiles.}

The $\mathrm{T}_{\mathrm{e}}$ measurements at all radii show oscillations at the same frequency, the different channels are either in phase or in anti-phase. Fig. 5 shows $\mathrm{T}_{\mathrm{e}}(\mathrm{R})$ at the maximum and minimum of an oscillation. The mode activity is clearly visible. The oscillations in the region $0.60<\mathrm{R}<0.64 \mathrm{~m}$ are in phase with the oscillations at $0.80<\mathrm{R}<0.88 \mathrm{~m}$ and are therefore associated with an even m-number, the obvious candidate being the $(\mathrm{m}, \mathrm{n})=(2,1)$ mode. The oscillations in the region $0.64<\mathrm{R}<0.70 \mathrm{~m}$ have opposite phase from the oscillations at $0.74<\mathrm{R}<$ 0.80 , and are associated with the $(\mathrm{m}, \mathrm{n})=(1,1)$ mode.

The same frequency is measured for all modes. This indicates that the modes are coupled and have the same toroidal mode number $(n=1)$, assuming the mode rotation is dominantly toroidal. Analysis of the $\mathrm{T}_{\mathrm{e}}$ profiles shows that the modes are coupled in such a way that the O-point of the $m=1$ mode locks to the $\mathrm{X}$ point of the $\mathrm{m}=2$ mode at the high field side.

The large amplitude of the oscillations between $\mathrm{R}=0.78$ and $\mathrm{R}=0.82 \mathrm{~m}$ is attributed to deformation of the flux surfaces due to the presence of the locked $m=1$ and $m=2$ islands, rather than that the island separatrix penetrates so far inwards.

The interferometer data are averaged over one oscillation period and Abel-inverted to yield the $\mathrm{n}_{\mathrm{e}}$ profile in the midplane around $t=142 \mathrm{~ms}$ (Fig. 5). To study the effect of the mode oscillations, the measured phase (proportional to the line integrated density) of all channels is plotted versus time and radius while subtracting the average phase (Fig. 6a). The effect of the $\mathrm{m}=2$ mode can be seen clearly. The central $\mathrm{m}=1$ mode does not show up clearly in the line-integrated interferometer data. To evaluate the perturbation of the density profile, the measurements have been simulated numerically with an $\mathrm{m}=2$ mode structure (a Gaussian in the radial and poloidal directions) at a chosen minor radius, with specified radial and poloidal width, and rotation frequency. These parameters are varied to make the simulated measurements match the experimental data.

The result of this procedure is shown in the second plot of Fig. 6b. The centre of the perturbation is found to be located at $\mathrm{r}=13.5 \mathrm{~cm}$, in fair agreement with the $\mathrm{r}(\mathrm{q}=2)=13 \mathrm{~cm}$ determined from the q-profile. The radial 1/e half-width is $3 \mathrm{~cm}$, which is consistent with the island width $\mathrm{w}=6.4 \mathrm{~cm}$ determined from the magnetic measurements. Finally, the poloidal 1/e halfwidth of $\approx 1 \mathrm{rad}$ corresponds to the lowest harmonic of an $\mathrm{m}=2$ mode.

The amplitude of the simulations is scaled to reproduce the actual measured oscillation amplitudes, this yields a local $n_{e}$ oscillation amplitude of $n_{e}(\max )$ $\mathrm{n}_{\mathrm{e}}(\min )=1.3 \cdot 10^{19} \mathrm{~m}^{-3}$, superimposed on an average $\mathrm{n}_{\mathrm{e}} \approx 3 \cdot 10^{19} \mathrm{~m}^{-3}$.

3.2.4. The phase relation of the $B_{\theta}, n_{e}$, and $T_{e}$ oscillations, identification of the island O-point.

In Fig. 7 the signals $B_{\theta}(\theta=0), T_{e}(R=0.843$ $\mathrm{m}), \mathrm{T}_{\mathrm{e}}(\mathrm{R}=0.868 \mathrm{~m}), \overline{\mathrm{n}}_{\mathrm{e}}(\mathrm{R}=0.594 \mathrm{~m})$ and $\overline{\mathrm{n}}_{\mathrm{e}}(\mathrm{R}=$ $0.864 \mathrm{~m}$ ) are compared. The $\mathrm{T}_{\mathrm{e}}$ and $\mathrm{B}_{\theta}$ measurements are taken at the same toroidal position, while the interferometer is located at $120^{\circ}$ toroidal angle, for which the phase can be corrected as shown in the figure. With the mode rotating in the electron drift direction, pure toroidal rotation will give the $\mathrm{T}_{\mathrm{e}}$ and $\mathrm{B}_{\theta}$ measurements a $120^{\circ}$ phase lag with respect to the $n_{e}$ signal.

In all discharges investigated the $\mathrm{T}_{\mathrm{e}}$ and $\mathrm{B}_{\theta}$ oscillations are in phase. Since the shear is positive, the island O-point corresponds to a negative perturbation of the local current density and hence to a minimum in $\mathrm{B}_{\theta}$ measured outside the plasma. Thus, the $\mathrm{O}$-point is a relatively cold region for the $\mathrm{m}=2$ island.

There remains a small phase difference between the $\mathrm{T}_{\mathrm{e}}$ and $\mathrm{n}_{\mathrm{e}}$ oscillations after correcting for the $120^{\circ}$ of toroidal separation between the diagnostics. In Fig. $7 \mathrm{a}$ the $\mathrm{n}_{\mathrm{e}}$ oscillation leads by some $40^{\circ}$. In 3 other discharges, with otherwise the same phenomenology but higher sampling rate of the diagnostics, the $\mathrm{n}_{\mathrm{e}}$ oscillation lags by $0^{\circ}, 20^{\circ}$ and $25^{\circ}$, respectively. The measured phase differences may partly be due to the limited resolution of the phase measurement. Also, by finite poloidal rotation preceding the minor disruption some phase difference may be induced. The general observation is that the $\mathrm{B}$, $\mathrm{T}_{\mathrm{e}}$ and $\mathrm{n}_{\mathrm{e}}$ oscillations are all in phase, so that the $\mathrm{O}$ point is both colder and of lower density than the Xpoint.

\subsubsection{The gradient of $T_{e}$ across the island.}

In Fig. 5 the $\mathrm{T}_{\mathrm{e}}$ profile in the island $\mathrm{X}$ - and $\mathrm{O}$-points is shown. While it is clear that the O-point is cooler than the X-point, no flattening of the profile is observed. The same is evidenced by the contour-plots for both $\mathrm{T}_{\mathrm{e}}$ and $\mathrm{n}_{\mathrm{e}}$. The question is, could there be a flat spot that goes unnoticed? As indicated in Fig. 5, the island (as computed from the magnetic measurements) covers 3 channels of the ECE diagnostic, at $\mathrm{R}=0.81,0.84$, and $0.87 \mathrm{~m}$. At all three positions, $\mathrm{T}_{\mathrm{e}}$ is lower in the $\mathrm{O}$ point than in the X-point. There is no form of $T_{e}(R)$ featuring a flat area of the size of the island which is compatible with these measurements. Even if the O- 
point lies at a smaller minor radius than the $\mathrm{X}$-point, so that no phase reversal of the $\mathrm{T}_{\mathrm{e}}$ oscillations need be observed, the ECE channels at $\mathrm{R}=0.84$ and 0.87 should measure the same $T_{e}$ if there is no gradient across the island. This holds even when the width of the island is reduced due to the toroidicity.

As it is, the measured gradient across the island is hardly diminished compared to the X-point. Perhaps even more illustrative is the contour plot of $\mathrm{T}_{\mathrm{e}}$, in which the typical flux contours of a magnetic island are sketched. Taking the measurements at face value, the $\mathrm{T}_{\mathrm{e}}$-contours follow the flux-surfaces down to a temperature of $\approx 250 \mathrm{eV}$, while below that the two become completely disconnected. Below we investigate the possibility that the divergence of flux and $\mathrm{T}_{\mathrm{e}}$ contours is not an artifact of the measurement, but due to transport inside the island.

Also on the $n_{e}$ profile no flattening across the island is observed, and since the $\mathrm{n}_{\mathrm{e}}$ and $\mathrm{T}_{\mathrm{e}}$ oscillations are in phase, this implies that the electron pressure $\left(\mathrm{p}_{\mathrm{e}}\right)$, too, has a gradient across the island. Whereas the $\mathrm{T}_{\mathrm{e}}$ gradient might be explained on the basis of transport inside the island, a direct consequence of $\nabla p_{e}$ across the island is that $\mathrm{p}_{\mathrm{e}}$ is not constant on a flux surface. This probably implies that the total pressure $\mathrm{p}$, also, is not constant on a flux surface, and thus the magnetic island structure is not a solution of the standard MHD equilibrium equation $\nabla \mathrm{p}=\mathbf{j} \times \mathbf{B}$.

\section{MODELLING TRANSPORT ACROSS AN ISLAND.}

We consider the network shown in Fig. 8. This network models the heat flow across and along flux surfaces in the island. The ratio of the local parallel and cross-field heat resistances is given by the parameter $\alpha$, which may be a function of the minor radius inside the island. To arrive at a continuous solution, we let the number of shells (N) go to infinity. A general treatment of the network is given in Appendix A. The model allows the computation of the $\mathrm{T}_{\mathrm{e}}$ distribution and the net heat flow across the island, when a $\mathrm{T}_{\mathrm{e}}$ difference $2 \Delta \mathrm{T}_{\mathrm{e}}$ is applied to the boundaries. The spatial coordinate $\rho$ will be used to parametrize the position in the island, with $\rho=1$ at the high $T_{e}$ side of the island, $\rho=0$ at the magnetic axis, and $\rho=-1$ at the low $\mathrm{T}_{\mathrm{e}}$ side. The present network is symmetric with respect to $\rho=0$. In the following we will omit the subscript 'e' for clarity of notation.

We first compute for which value of $\alpha$ a flat spot in $\mathrm{T}$ is produced. Analytic results for two illustrative cases, $\alpha=$ constant and $\alpha \propto \rho^{2}$ are given by

$$
\alpha(\rho)=\alpha_{0} \quad \frac{T(\rho)-T(0)}{\Delta T}=\frac{\sinh \left(\rho / \sqrt{\alpha_{0}}\right)}{\sinh \left(1 / \sqrt{\alpha_{0}}\right)}
$$

$$
\begin{aligned}
& \alpha(\rho)=\alpha_{0} \rho^{2} \quad \frac{T(\rho)-T(0)}{\Delta T}=\rho^{\gamma} \\
& \gamma=\frac{1}{2}\left\{1+\sqrt{1+4 / \alpha_{0}}\right\}
\end{aligned}
$$

The replacement resistance for a flow across the network is given by

$$
\begin{aligned}
& \alpha(\rho)=\alpha_{0} \\
& \mathrm{R}_{\text {repl }}=\mathrm{R}_{0} \sqrt{\alpha_{0}} \tanh \left(1 / \sqrt{\alpha_{0}}\right)
\end{aligned}
$$

where $\mathrm{R}_{0}$ denotes the resistance of the network for $\alpha_{0} \rightarrow \infty$ (infinite parallel resistivity). In Fig. $9 a$ these results are shown for a few values of $\alpha_{0}$. $R_{\text {repl }}$ is shown as a function of $\alpha_{0}$ in Fig. 9b. Clearly a flat spot in $T(\rho)$ is only produced if $\alpha_{0}$ is smaller than some critical value $\alpha_{\text {crit }}$. Arbitrarily defining the occurrence of significant flattening by $\mathrm{T}(0.5)=$ $0.1 \times \mathrm{T}(1)$, we find $\alpha_{\text {crit }}=0.048$ in the case of uniform $\alpha$, and $\alpha_{\text {crit }}=0.13$ for the parabolic $\alpha$ profile. In the following we will use $\alpha_{\text {crit }}=0.1$ as an average value.

Next, we have to link the parameter $\alpha$ to plasma diffusivities along and across the field. In the simple analog sketched above, we consider a toroidal shell of the island, with - normalized - radius $\rho$ and thickness $d \rho$. It is clear that the parallel and perpendicular "resistances" (measured in terms of transport timescales) relate to the plasma diffusivities as follows:

$$
\begin{aligned}
& \mathrm{R}_{/ /}=\frac{1}{\mathrm{n} \chi_{/ /}} \frac{1_{/ /}}{\mathrm{A}_{/ /}} \\
& \mathrm{R}_{\perp}=\frac{1}{\mathrm{n} \chi_{\perp}} \frac{1_{\perp}}{\mathrm{A}_{\perp}}
\end{aligned}
$$

where 1 is the path length and $\mathrm{A}$ is the area. Assuming $\left(\chi_{\perp} / \chi_{/ /}\right) \ll 1$, and that the field lines in the island make only a small angle with the magnetic axis of the island, we have

$$
\begin{array}{ll}
1_{/ /}=\mathrm{m} \pi \mathrm{Rq}^{*} & \mathrm{~A}_{/ /} \approx \rho \mathrm{wd} \rho \frac{2 \pi \mathrm{r}}{\mathrm{m}} \frac{2}{\mathrm{q}^{*}} \\
1_{\perp}=\mathrm{wd} \rho & \mathrm{A}_{\perp} \approx 4 \pi^{2} \mathrm{rR} \rho
\end{array}
$$

where $\mathrm{q}^{*}$ denotes the local q-value in the island, giving the helicity of the field lines along the island flux tube. For $\alpha$ the following expression is obtained:

$$
\alpha=\frac{\mathrm{R}_{/ /}}{\mathrm{R}_{\perp}}(\mathrm{d} \rho)^{2}=\frac{\chi_{\perp}}{\chi_{/ /}}\left(\frac{\mathrm{m} \pi \mathrm{Rq}^{*}}{\mathrm{w}}\right)^{2}=\frac{\tau_{/ /}}{\tau_{\perp}}
$$


where $\tau_{/ /}=\left(\mathrm{m} \pi \mathrm{Rq}^{*}\right)^{2} / \chi_{/ /}$and $\tau_{\perp}=\mathrm{w}^{2} / \chi_{\perp}$ are the parallel and perpendicular transport times within the island, respectively. In Appendix B a more detailed derivation of eq. (9) is given.

As was shown above, a flat spot in the $\mathrm{T}$ profile is produced for $\alpha<0.1$. From eq. (9) it is clear that, although $\left(\chi_{\perp} / \chi_{/ /}\right)$is usually a very small number in tokamaks, the numerical factor in eq. (9) multiplying this term can be very large, so that it cannot be concluded that $\alpha<0.1$ without careful consideration. Below we discuss the three critical parameters in eq. (9), $\mathrm{q}^{*}, \chi_{\perp}$, and $\chi_{/ /}$.

At the separatrix $\mathrm{q}^{*}$ becomes infinite, but it is not clear what the profile of $\mathrm{q}^{*}$ in the island is, since the current distribution in the island is unknown. For any toroidal current, the cylindrical $\mathrm{q}$ is given by

$$
\mathrm{q}_{\mathrm{cyl}}=\frac{\mathrm{l}_{\theta}^{2}}{\mu_{0} \delta \mathrm{I}} \frac{\mathrm{B}_{\phi}}{\mathrm{l}_{\phi}}
$$

where $1_{\theta}$ is the length of a circuit along a flux surface around the current channel in the poloidal plane, $\delta \mathrm{I}$ the current flowing through the surface surrounded by the circuit, and $l_{\phi}$ the length of the flux tube in the toroidal direction. For the island, $1_{\theta} \approx 4 \pi \mathrm{r} / \mathrm{m}$ and $1_{\phi}=2 \pi \mathrm{Rm}$, so an estimate for $\mathrm{q}^{*}$ just inside the separatrix is given by

$$
\mathrm{q}^{*}=\frac{8 \pi \mathrm{r}^{2} \mathrm{~B}_{\phi}}{\mu_{0} \mathrm{~m}^{3} \delta \mathrm{I} R}
$$

where $\delta$ I denotes the incremental current associated with the island O-point, as before. Inserting the typical values for the $m=2$ island under study, $q^{*} \approx 100$ is found. The profile of $\mathrm{q}^{*}$ could be flat if the current density is uniform in the island, but if the current profile in the island is peaked, the central $\mathrm{q}^{*}$ is correspondingly lower. The two analytic cases given above, with $\alpha$ uniform or parabolic, are representative of a fairly wide range of possible $\mathrm{q}^{*}$ profiles.

Further insight is obtained when the estimate for $\mathrm{q}^{*}$ (eq. (10)) is expressed in terms of the width of the island (using eqs. (1), (2) and (7)):

$$
\mathrm{q}^{*}=32 \pi \frac{\mathrm{r} \mathrm{q}^{2}}{\mathrm{~m}^{3} \mathrm{w}^{2} \mathrm{q}^{\prime}}
$$

which, inserted in eq. (9), yields

$$
\alpha=\frac{\chi_{\perp}}{\chi_{/ /}}\left(\frac{\mathrm{r} \mathrm{R} \mathrm{q}^{2}}{\mathrm{q}^{\prime} \mathrm{w}^{3} \mathrm{~m}^{2}}\right)^{2}\left(32 \pi^{2}\right)^{2}
$$

Using $\mathrm{q}=\mathrm{m}$ and assuming a parabolic $\mathrm{q}$-profile, this can be further reduced to

$$
\alpha=\frac{\chi_{\perp}}{\chi_{/ /}}\left(\frac{16 \pi^{2}}{q(a)-q(0)}\right)^{2}\left(\frac{R}{a}\right)^{2}\left(\frac{a}{w}\right)^{6}
$$

Note the extreme sensitivity of $\alpha$ to $a / w$. For the parallel conductivity we use the classical expression

$$
\chi_{/ /}=v_{e}^{2} \tau_{e}=1.210^{4} \frac{\mathrm{T}_{\mathrm{e}}^{5 / 2}}{\mathrm{n}_{\mathrm{e}} \mathrm{Z}_{\mathrm{eff}} \ln \Lambda}\left[\mathrm{m}^{2} / \mathrm{s}\right]
$$

where $\mathrm{T}_{\mathrm{e}}$ is in $\mathrm{eV}$ and $\mathrm{n}_{\mathrm{e}}$ in $10^{19} \mathrm{~m}^{-3}$. For the crossfield diffusivity we will first assume that inside the island transport is anomalous like elsewhere. As a typical value for the outer part of the plasma we take

$$
\chi_{\perp}(\text { anom })=10 \mathrm{~m}^{2} / \mathrm{s}
$$

However, it can be argued - following the KAMtheorem - that inside the island the flux surfaces are close to perfect, resulting in a strongly reduced transport. We will come back to this possibility later in the discussion.

Inserting typical values for the $\mathrm{m}=2$ island under consideration yields $(\mathrm{R} / \mathrm{w}=15, \mathrm{~T} \backslash \mathrm{S} \backslash \mathrm{DO} 3(\mathrm{e})=200 \mathrm{eV}$, $\left.\mathrm{n}_{\mathrm{e}}=310^{19} \mathrm{~m}^{-3}, \mathrm{Z}_{\mathrm{eff}}=3, \mathrm{q}^{*}=100\right)$ :

$$
\begin{aligned}
& \chi_{/ /}=510^{7} \mathrm{~m}^{2} / \mathrm{s} \\
& \alpha \approx \frac{\chi_{\perp}}{\chi_{/ /}} 510^{7} \approx 10>0.1
\end{aligned}
$$

which implies no flattening of $\mathrm{T}_{\mathrm{e}}(\mathrm{r})$. It is seen that if the cross-field transport inside the island is anomalous and comparable to that in the ambient plasma, under no circumstances can one expect a flat spot in the temperature profile. In general, the fact that sometimes no flattening is observed can be simply explained by the (relative) ineffectiveness of the transport along the field lines.

The fact that in some experiments $[3,5,7,8$, 9] flat spots have been observed in $(\mathrm{m}, \mathrm{n})=(2,1)$ islands, whereas in others $[4,6,10]$ only reduced gradients or no flat spots at all have been observed, may serve as an indication that $\alpha$ in general is close to its critical value, so that the occurrence of flat spots depends rather sensitively on specific discharge conditions. This could also explain why, in our case, in the higher $\mathrm{T}_{\mathrm{e}}$ region the temperature contours follow the flux contours quite well, while in the lower $\mathrm{T}_{\mathrm{e}}$ part of the island the two are completely uncorrelated. Taking $\alpha=\alpha_{\text {crit }}$ yields an estimate of $\chi_{\perp}$ inside the magnetic island:

$$
\alpha=\alpha_{\text {crit }} \quad \Rightarrow \quad \chi_{\perp}=0.1 \pm 0.06 \mathrm{~m}^{2} / \mathrm{s}
$$


This value is somewhat larger than the neoclassical value, but of the same order of magnitude. The large error bar is the result of the strong dependence on the width of the island.

\section{DISCUSSION AND CONCLUSIONS}

We have presented experimental evidence that there may exist $\mathrm{T}_{\mathrm{e}}$ and $\mathrm{n}_{\mathrm{e}}$ gradients across magnetic islands, and we have shown that a simple consideration of parallel and cross-field transport indicates that such gradients can be maintained, depending on the width of the island and the local values of $\mathrm{T}_{\mathrm{e}}, \mathrm{n}_{\mathrm{e}}$ and $\mathrm{Z}_{\mathrm{eff}}$.

A consistent picture is obtained by assuming that the cross-field transport inside the island is close to neoclassical. In this case, the total heat resistance across the island is large, so that only a small fraction of the total heat flux can be conducted through the island O-point. Hence, the main heat flux must be conducted through the X-points.

A further consequence is that, with vanishing mode rotation with respect to the fluid, the energy and particle balance in the island is well insulated from the surrounding plasma. In that case the temperature in the island is entirely determined by the local source and sink terms. In the absence of particle sinks, the particle confinement in the island will be very good. This conclusion agrees well with the observation of the snake in JET [2]. It is likely that the density inside the island is determined at the time of birth of the island. Whether the O-point is a low or a high density region then depends on the evolution of the density in the ambient plasma. This may explain that low density as well as high density islands have been observed [15].

From eq. (13) it is clear that the value of $\alpha$ is a very strong function of the width of the island. Thus, it is to be expected that a slowly growing $m=2$ island will quite suddenly reach the condition $\alpha<\alpha_{\text {crit }}$ for which flattening of the $T_{e}$ profile occurs. The violent, sawtoothing, behaviour of $\mathrm{T}_{\mathrm{e}}(\mathrm{r})$ observed in quasi stationary $\mathrm{m}=2$ modes in JET [3], in which a sudden flattening of the $T_{e}$ profile around the $q=2$ radius was followed by a gradual restoration of the $\mathrm{T}_{\mathrm{e}}$ gradient, and the sudden flattening of $\mathrm{T}_{\mathrm{e}}(\mathrm{r})$ observed in $\mathrm{m}=2$ modes in TEXTOR [6], followed by a heatpulse, might in part be due to this transport phenomenon. This sudden flattening would then be caused by $\alpha$ being driven below the threshold value, which can occur on a short time scale even when the increase of $w$ is comparatively slow.

Mode structure rotation with respect to the fluid is capable of (partially) obliterating flat spots. This effect commonly occurs when MHD modes in the outer regions of the plasma are locked to modes located in the centre, whereas the fluid rotation velocity decreases towards the plasma edge due to friction. The importance of this effect can be estimated by comparing the characteristic relative rotation time, $\tau_{\text {rot }}$ $=1 / \mathrm{f}_{\text {rot }}$, with the characteristic time for transport along the field lines inside the island, $\left\langle\tau_{/ /}\right\rangle \approx$ $\mathrm{C}\left(\mathrm{m} \pi \mathrm{Rq}^{*}\right)^{2} / \chi_{/ /}$, where $\mathrm{C}$ is a constant depending on the shape of the $\mathrm{q}^{*}$ profile within the island, $\mathrm{C} \approx 0.2$, and $<>$ means averaging over the island region (cf. eq. (9)). This mechanism, capable of sustaining both $T_{e}$ and $\mathrm{n}_{\mathrm{e}}$ gradients in the $\mathrm{O}$-point region by continually importing them from the $\mathrm{X}$-point region, has previously been reported by Jaenicke (Wendelstein VIIA) [10]. It may also be the explanation for the observation on TEXTOR [6] of a flat spot in $\mathrm{T}_{\mathrm{e}}$ in a wall-locked $(\mathrm{m}, \mathrm{n})=(2,1)$ island that disappears when the island unlocks and starts rotating, and reappears when the island locks again.

Wesson [4] reports on observations of an $(\mathrm{m}, \mathrm{n})=(2,1)$ island at low $\mathrm{T}_{\mathrm{e}}\left(\mathrm{T}_{\mathrm{e}} \approx 400 \mathrm{eV}, \# 11051\right)$. While the mode is rotating (at $f=700 \mathrm{~Hz}$ ) no clear flat spot is seen, only a reduction of the $\mathrm{T}_{\mathrm{e}}$ gradient. As the mode locks, a flat spot becomes visible of $w\left(T_{\mathrm{e}}\right) \approx 8$ $\mathrm{cm}$. This width is somewhat less than the width expected from magnetic measurements. Temperature profiles within the island as predicted by our model when $\alpha$ is close to critical (see Fig. 9a) may explain the latter. During mode rotation the gradients may be enhanced by the mechanism explained above.

In summary, the observation of flattenings in the $T_{e}$ profiles at high $T_{e}$ with widths as calculated by magnetic measurements are explained by $\alpha<\alpha_{\text {crit }}$ (parallel transport dominates perpendicular transport within the island). Observations of the absence of flat spots are explained by $\alpha \gg \alpha_{\text {crit }}$, reduced gradients or flat spots with widths smaller than the widths calculated from magnetics by $\alpha \approx \alpha_{\text {crit. }}$. The latter is commonly observed at low $\mathrm{T}_{\mathrm{e}}$ with reduced parallel transport. Finally, mode rotation with respect to the plasma may reduce observed island widths in the $T_{e}$ profiles when the characteristic time for transport parallel to the field lines is smaller than the characteristic time for penetration of the $\mathrm{X}$-point profiles into the O-point region.

Not only a $\mathrm{T}_{\mathrm{e}}$ gradient, but also a $\mathrm{n}_{\mathrm{e}}$ gradient is maintained across the island, which can be explained as a transport phenomenon in analogous fashion. One severe consequence is that pressure is no longer constant on a flux surface, so that the equilibrium does not satisfy $\nabla \mathrm{p}=\mathbf{j} \times \mathbf{B}$. This implies that convective fluxes must be invoked to maintain the equilibrium. This would in turn change the transport analysis. A consistent treatment of the island equilibrium including the transport aspects is outside the scope of this paper. However, two remarks relevant to this problem can be made. Firstly, because of the very long connection length the characteristic time for equilibration of a pressure gradient along a field line is quite long: assuming that the plasma flows towards the low pressure region with ion acoustic velocity this 
characteristic time is several milliseconds. This flow could be further hampered by friction with neutrals, of which the density may be quite significant in this part of the plasma. Secondly, the rotation of the fluid with respect to the magnetic island may help maintain the flow, and hence the pressure gradient.

A second possibility to explain the observations not discussed so far is that there is no magnetic island at all, but a kink deformation located at the $\mathrm{q}=2$ surface. Such a deformation would also give the $\mathrm{n}_{\mathrm{e}}$ and $\mathrm{T}_{\mathrm{e}}$ contours as observed, with $\mathrm{n}_{\mathrm{e}}$ and $\mathrm{T}_{\mathrm{e}}$ always in phase. The problem is of course that the internal $\mathrm{m}=2 \mathrm{kink}$ is stable. However, one might argue that the cold plasma outside the $\mathrm{q}=2$ surface becomes so resistive that it starts to act as a limiter, reducing the effective plasma radius to $\mathrm{r}(\mathrm{q}=2)$. Whether in such a situation a kink or a tearing mode becomes unstable depends critically on how large the resistivity of the plasma outside the $\mathrm{q}=2$ surface becomes. So far no clear answer to this problem could be given.

\section{APPENDIX A:}

\section{SIMULATION OF HEAT FLOW IN MAGNETIC ISLAND BY MEANS OF A RESISTANCE NETWORK}

In Fig. A.1 a network of resistors is shown. The resistors $r_{/ /}$and $r_{\perp}$ have values that may depend on their position or other quantities. We introduce a poloidal coordinate system $(\rho, \theta)$, where $\rho$ is a radial coordinate, $\rho=0$ in the centre and $\rho=1$ at the boundary and $\theta$ is a poloidal coordinate, $0 \leq \theta<2 \pi$. An externally imposed voltage $\mathrm{V}(\rho=1, \theta)$, which we take to be the analogue of the temperature of the plasma surrounding the island, will result in a voltage distribution $\mathrm{V}(\rho, \theta)$ in the network, which is analogous to the temperature distribution inside the island. The amplitude of $\mathrm{V}$ (imposed externally) will not affect the shape of the distribution, and neither will the absolute values of the resistors: only the ratio of $r_{/ /}$and $r_{\perp}$ in each point (analogous to the inverse ratio of thermal conductivities parallel and perpendicular to the field lines) influence the shape of the voltage (or temperature) distribution.

Referring again to Fig. A.1, the grid is defined by $\rho_{i}=\mathrm{i} / \mathrm{N}_{\rho}\left(\mathrm{i}=1, \ldots, \mathrm{N}_{\rho}\right)$ and $\theta_{\mathrm{j}}=2 \pi(\mathrm{j}-1) /$ $\mathrm{N}_{\theta}\left(\mathrm{j}=1, \ldots, \mathrm{N}_{\theta}\right)$, where $\mathrm{N}_{\rho}$ and $\mathrm{N}_{\theta}$ are the number of radial and poloidal grid points, respectively. The voltage distribution, given the boundary conditions, should relax to a situation where each internal point of the network receives zero net current. This implies, for each internal node $\left(\rho_{\mathrm{i}}, \theta_{\mathrm{j}}\right)$ :

$$
\begin{aligned}
& \mathrm{r}_{\perp}^{-1}\left[\mathrm{~V}\left(\rho_{\mathrm{i}+1}, \theta_{\mathrm{j}}\right)+\mathrm{V}\left(\rho_{\mathrm{i}-1}, \theta_{\mathrm{j}}\right)-2 \mathrm{~V}\left(\rho_{\mathrm{i}}, \theta_{\mathrm{j}}\right)\right]+ \\
& \mathrm{r}_{/ /}^{-1}\left[\mathrm{~V}\left(\rho_{\mathrm{i}}, \theta_{\mathrm{j}+1}\right)+\mathrm{V}\left(\rho_{\mathrm{i}}, \theta_{\mathrm{j}-1}\right)-2 \mathrm{~V}\left(\rho_{\mathrm{i}}, \theta_{\mathrm{j}}\right)\right]=0
\end{aligned}
$$

which, in the case of $\mathrm{N}_{\rho}>1$ and $\mathrm{N}_{\theta}>>1$, can be written (defining $\Delta \rho=1 / \mathrm{N}_{\rho}$ and $\Delta \theta=2 \pi / \mathrm{N}_{\theta}$ ):

$$
\frac{\partial^{2} \mathrm{~V}}{\partial \rho^{2}} \frac{\Delta \rho^{2}}{\mathrm{r}_{\perp}}+\frac{\partial^{2} \mathrm{~V}}{\partial \theta^{2}} \frac{\Delta \theta^{2}}{\mathrm{r}_{/ /}}=0
$$

We introduce a parameter $\alpha$, that defines the ratio of $r_{/ /}$ and $\mathrm{r}_{\perp}$ :

$$
\alpha=\frac{\mathrm{r}_{/ /}}{\mathrm{r}_{\perp}} \frac{\mathrm{N}_{\theta}^{2}}{16 \mathrm{~N}_{\rho}^{2}}
$$

In Appendix B we will show how $\alpha$ relates to the ratio of thermal conductivities. Substituting in eq. (A.2) yields:

$$
\frac{\partial^{2} V}{\partial \rho^{2}}+\frac{\partial^{2} V}{\partial \theta^{2}} \frac{\pi^{2}}{4 \alpha}=0
$$

The equation for the voltage distribution in the network. If $\alpha=\alpha(\rho)$ and $\mathrm{V}(\rho, \theta)=f(\rho) g(\theta)$, then eq. (A.4) reduces to:

$$
-\frac{4 \alpha}{\pi^{2}} \frac{1}{f} \frac{\partial^{2} f}{\partial \rho^{2}}=\frac{1}{g} \frac{\partial^{2} g}{\partial \theta^{2}}=\mathrm{m}^{2}
$$

where $\mathrm{m}$ is a constant (not related to the poloidal mode number). The solution for $g$ is $g_{\mathrm{m}}=\mathrm{A}_{\mathrm{m}} \cos (\mathrm{m} \theta)+\mathrm{B}_{\mathrm{m}} \sin (\mathrm{m} \theta)$. We may set $f(1)=1$ without loss of generality, so $A_{m}$ and $B_{m}$ can be found from a Fourier transform of the boundary condition $\mathrm{V}(\rho=1, \theta)$.

There are two cases of functional dependence of $\alpha$ on $\rho$ for which the equation for $f$ can easily be solved analytically:

$$
f_{\mathrm{m}}(\rho)=\sinh \left\{\frac{\rho \mathrm{m} \pi}{2 \sqrt{\alpha_{0}}}\right\} \sinh ^{-1}\left\{\frac{\mathrm{m} \pi}{2 \sqrt{\alpha_{0}}}\right\}
$$

for $\alpha(\rho)=\alpha_{0}$ (i.e. constant) and

$$
f_{\mathrm{m}}(\rho)=\rho^{\gamma_{\mathrm{m}}, \gamma_{\mathrm{m}}}=\frac{1}{2}+\frac{1}{2} \sqrt{1+\frac{(\mathrm{m} \pi)^{2}}{\alpha_{0}}}
$$

for $\alpha(\rho)=\alpha_{0} \rho^{2}$. Finally, we give the equation for the one-dimensional network with $\mathrm{N}_{\theta}=2$, for which eq. (A.4) must be replaced by:

$$
\frac{\partial^{2} \mathrm{~V}}{\partial \rho^{2}}+\frac{\mathrm{V}}{\alpha}=0
$$

which was deduced from eq. (A.1) using the antisymmetry $\mathrm{V}(-\rho)=-\mathrm{V}(\rho)$. The $\theta$-coordinate no longer appears and $\alpha=\alpha(\rho)$. The solutions to eq. (A.6) are $\mathrm{V}(\rho)=f_{\mathrm{m}}(\rho)$ with $\mathrm{m}=2 / \pi$. 
The replacement resistance of the whole network can be computed from:

$$
\mathrm{R}_{\mathrm{repl}}=\mathrm{R}_{0}\left(\frac{\mathrm{V}}{\frac{\partial \mathrm{V}}{\partial \rho}}\right)_{\rho=1}
$$

where $R_{0}$ is the resistance of the network in the case that $r_{/ /}$is infinite everywhere.

\section{APPENDIX B:}

\section{DERIVATION OF $\alpha$ AS A FUNCTION OF HEAT CONDUCTIVITIES}

The assumed flux surface geometry inside a magnetic island is shown in Fig. B.1. We label the flux surfaces with a parameter $\rho$ that is 0 in the centre of the island and 1 at the separatrix. We propose to model the heat flow in the island by means of a one-dimensional resistance network $\left(\mathrm{N}_{\theta}=2\right.$, see Appendix A). There is no difference in principle with the two-dimensional network.

We consider a point on the inside of the island (i.e. at $\theta=\pi$ ). Heat flowing from this point can go in three directions:

1) Perpendicular to the flux surface $(\perp)$.

2) Parallel to the flux surface and parallel to the field lines $(/ /, / /)$

3) Parallel to the flux surface but perpendicular to the field lines $(/ /, \perp)$.

We will calculate the "heat resistance" (i.e. the inverse of the thermal conductivity) in all three directions separately, and combine them to find $\alpha$.

The distance $\mathrm{p}_{1}-\mathrm{p}_{2}$, measured along the flux surface (Fig. B.1) perpendicular to the field lines at the separatrix, we call y. The length of the island in toroidal direction, measured along the field lines, we call $\mathrm{x}$. Thus, taking into account that the island is positioned at minor radius $r$ :

$$
\begin{aligned}
& x=2 \pi R_{0} m \sin ^{-1}(\xi), \\
& y=\rho \frac{2 \pi r}{m} \sin (\xi)
\end{aligned}
$$

where $\mathrm{m}$ is the poloidal mode number of the island and $\xi$ is the pitch angle of the field lines at the separatrix: $\tan (\xi)=\mathrm{qR}_{0} / \mathrm{r}$, where $\mathrm{q}=\mathrm{m} / \mathrm{n}$ and $\mathrm{n}$ is the toroidal mode number of the island (usually, $\sin (\xi)$ will be close to 1 and may be omitted). This expression for $y$ assumes that the island width $\mathrm{w}$ is small compared to its poloidal extent $2 \pi \mathrm{r} / \mathrm{m}$.

The "heat resistance" is given by the inverse of the thermal conductivity $\chi$ multiplied by the length of the path over which transport is to take place (1) and divided by the surface area through which the heat flows (A). By analogy with the resistance model
(Appendix A) we divide the island into $\mathrm{N}_{\rho}$ layers, so that the width of each layer is $w /\left(2 \mathrm{~N}_{\rho}\right)$, where $w$ is the island width.

1) Perpendicular transport:

$$
\begin{aligned}
& \mathrm{r}_{\perp}=\frac{1_{\perp}}{\mathrm{n}_{\mathrm{e}} \chi_{\perp} \mathrm{A}_{\perp}}, \\
& 1_{\perp}=\frac{\mathrm{w}}{2 \mathrm{~N}_{\rho}}, \\
& \mathrm{A}_{\perp}=\mathrm{xy}
\end{aligned}
$$

where $\mathrm{n}_{\mathrm{e}}$ is the electron density.

2) Transport parallel to the flux surface and parallel to the field lines:

$$
\begin{aligned}
& \mathrm{r}_{/ /}^{/ /}=\frac{\mathrm{1}_{/ /}^{/ /}}{\mathrm{n}_{\mathrm{e}} \chi_{/ /} \mathrm{A}_{/ /}^{/ /}} \\
& \mathrm{l}_{/ /}^{/ /}=\frac{\mathrm{y}}{\cos (\zeta)}, \\
& \mathrm{A}_{/ /}^{/ /}=\frac{\mathrm{w}}{2 \mathrm{~N}_{\rho}} \times \cos (\zeta)
\end{aligned}
$$

where $\zeta$ is the angle of the field lines on the flux surface with respect to the field lines at the separatrix (see Fig. B.1): $\tan (\zeta)=q^{*} x /(2 y)$. The factor 2 appears because $y$ refers to a half poloidal transit only. $1_{1}$ is the path length to go from $p_{1}$ to $p_{2}$ along the field lines (Fig. B.1) and $\mathrm{q}^{*}$ is the local $\mathrm{q}$ within the island.

3) Transport parallel to the flux surface but perpendicular to the field lines:

$$
\begin{aligned}
& \mathrm{r}_{\perp}^{\prime /}=\frac{\mathrm{l}_{\perp}^{\prime /}}{\mathrm{n}_{\mathrm{e}} \chi_{\perp} \mathrm{A}_{\perp}^{\prime /}}, \\
& \mathrm{l}_{\perp}^{\prime /}=\frac{\mathrm{y}}{\sin (\zeta)}, \\
& \mathrm{A}_{\perp}^{\prime /}=\frac{\mathrm{w}}{2 \mathrm{~N}_{\rho}} \mathrm{x} \sin (\zeta)
\end{aligned}
$$

where $l_{1}$ is the path length to go from $\mathrm{p}_{1}$ to $\mathrm{p}_{2}$ perpendicular to the field lines (Fig. B.1). The total transport parallel to the flux surface has to surmount a resistance equal to $r_{/ /}$:

$$
\mathrm{r}_{/ /}=\frac{\mathrm{r}_{/ /} \mathrm{r}_{\perp} / /}{\mathrm{r}_{/ /}+\mathrm{r}_{\perp}^{/ /}}
$$

Using the definition of $\alpha$ given by eq. (A.3) with $\mathrm{N}_{\theta}=$ 2 , the above equations yield: 


$$
\alpha=\frac{\mathrm{y}^{2}}{\mathrm{w}^{2}}\left[\sin ^{2} \zeta+\frac{\chi_{/ 1}}{\chi_{\perp}} \cos ^{2} \zeta\right]^{-1}
$$

This formula can be simplified by the assumption $\chi_{/ /} / \chi_{\perp}>\tan ^{2} \zeta>1$ :

$$
\alpha=\frac{\chi_{\perp}}{\chi_{/ /}}\left[\frac{\mathrm{m} \pi \mathrm{R}_{0} \mathrm{q}^{*}}{\mathrm{w}}\right]^{2} \frac{1}{\sin ^{2}(\xi)}
$$

which can be simplified further when $\sin (\xi) \approx 1$ (implying that the helicity of the island is negligible).

\section{ACKNOWLEDGMENTS}

It is a pleasure to acknowledge the RTP-team for excellent operation of the machine and diagnostics. We are indebted to Dr. J. Timmermans for his reconstructions of the perturbation of the density, to Dr. G.T.A. Huysmans for valuable discussions concerning the stability of the internal kink mode, and to Dr. D.C. Schram for his critical reading of our manuscript. This work was performed under the Euratom-FOM association agreement, with financial support from NWO and Euratom.

\section{REFERENCES}

[1] E. Westerhof, P. Smeulders and N.J. Lopes Cardozo, Nucl. Fusion 29 (1989) 1056 - 1061

[2] A. Weller, A.D. Cheetham, A.W. Edwards, R.D. Gill, et al., Phys. Rev. Lett. 59 (1987) 2303

[3] J.A. Snipes, D.J. Campbell, P.S. Haynes, T.C. Hender, M. Hugon, P.J. Lomas, N.J. Lopes Cardozo, M.F.F. Nave, F.C. Schüller, Nucl. Fusion 28 (1988) 1085 - 1097

[4] J.A. Wesson, R.D. Gill, M. Hugon, F.C. Schüller, J.A. Snipes, D.J. Ward, et al., Nucl. Fusion 29 (1989) 641 - 666

[5] N.A. Salmon, High Spatial Resolution Temperature Measurements in the JET Plasma Using Electron Cyclotron Emission, Ph.D. Thesis, Department of Plasma Physics, The Blacket Laboratory, Imperial College of Science, Technology and Medicine, South Kensington, London, November 1989

[6] G. Waidmann and G. Kuang, Nucl. Fusion 32 (1992) $645-654$

[7] M.F.F. Nave, A.W. Edwards, K. Hirsch, M. Hugon, A. Jacchia, E. Lazzaro, H. Salzmann, P. Smeulders, Nucl. Fusion 32 (1992) 825 - 835

[8] E.D. Fredrickson, K. McGuire, A Cavallo, B. Grek, K.-I. Hattori, D. Johnson, Rev. Sci. Instrum. 59 (1988) 1797 - 1800
[9] C.X. Yu, D.L. Brower, S.J. Zhao, W.A. Peebles, N.C. Luhmann Jr., R.V. Bravenec, J.Y. Chen, H. Lin, Ch.P. Ritz, P.M. Schoch and X.Z. Yang, Phys. Fluids B 4 (1992) 381 - 385

[10] R. Jaenicke, W VII-A Team, Nucl. Fusion 28 (1988) 1737 - 1750

[11] M. Verreck, C.A.J. Hugenholtz, Proc. SPIE $7^{\text {th }}$ Joint Workshop on ECE and ECRH and IAEA Technical Committee Meeting (EC-7), Hefei, PR China, May 9-11 (1989), Collection of Papers, IAEA, Vienna (1990) 322-327

[12] A.C.A.P. van Lammeren, Electron and current density measurements on tokamak plasmas, Ph.D. Thesis, FOM-Instituut voor Plasmafysica "Rijnhuizen", Nieuwegein, The Netherlands (1991)

[13] R.B. White and D.A. Monticello, Phys. Fluids 20 (1977) 800 - 805

[14] F.C. Schüller, D.C. Schram, J. Konings, A.C.A.P. van Lammeren, J.C.M. Timmermans, M. Verreck and the RTP Team, in Contr. Fus. and Plasma Phys. (Proc. $18^{\text {th }}$ Eur. Conf. Berlin, 1991), Vol. 15C, Part IV, European Physical Society (1991) IV-185

[15] B.Ph. van Milligen, Analysis of equilibrium and topology of tokamak plasmas, Ph.D. Thesis, FOM-Instituut voor Plasmafysica "Rijnhuizen", Nieuwegein, The Netherlands (1991) 


\section{FIGURES}
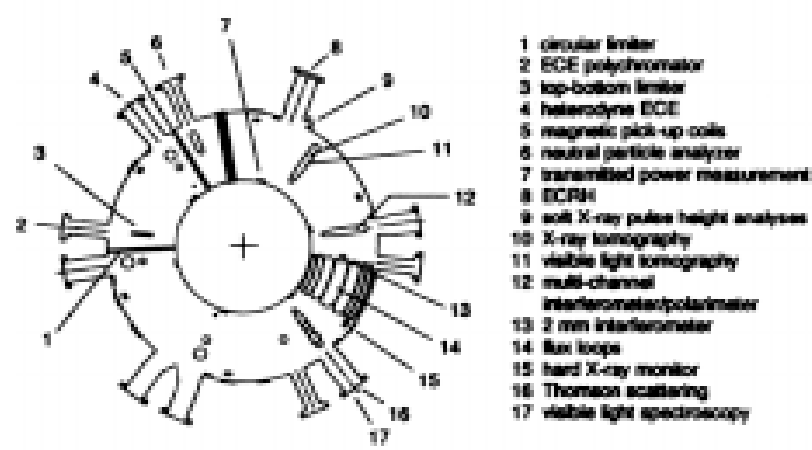

Fig. 1 Schematic showing the location of the main diagnostics at RTP. The tokamak is seen from the top. The plasma current and toroidal magnetic field are both in clockwise direction.

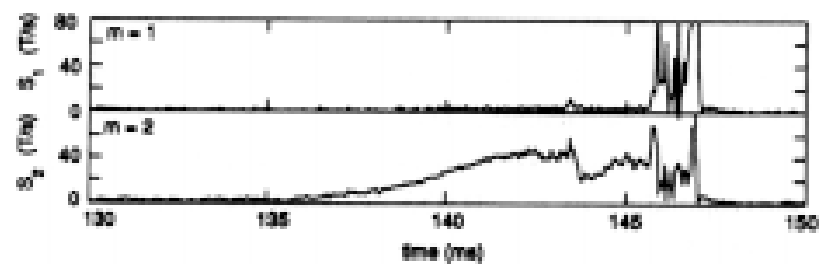

Fig. 2 MHD activity as computed by the method described in the text. The MHD activity is seen to commence at about $135 \mathrm{~ms}$ and grow to saturation at $142 \mathrm{~ms}$. The mode is dominantly $\mathrm{m}=2$. At about $144 \mathrm{~ms}$ a minor disruption occurs, followed by a major disruption at $147 \mathrm{~ms}$.

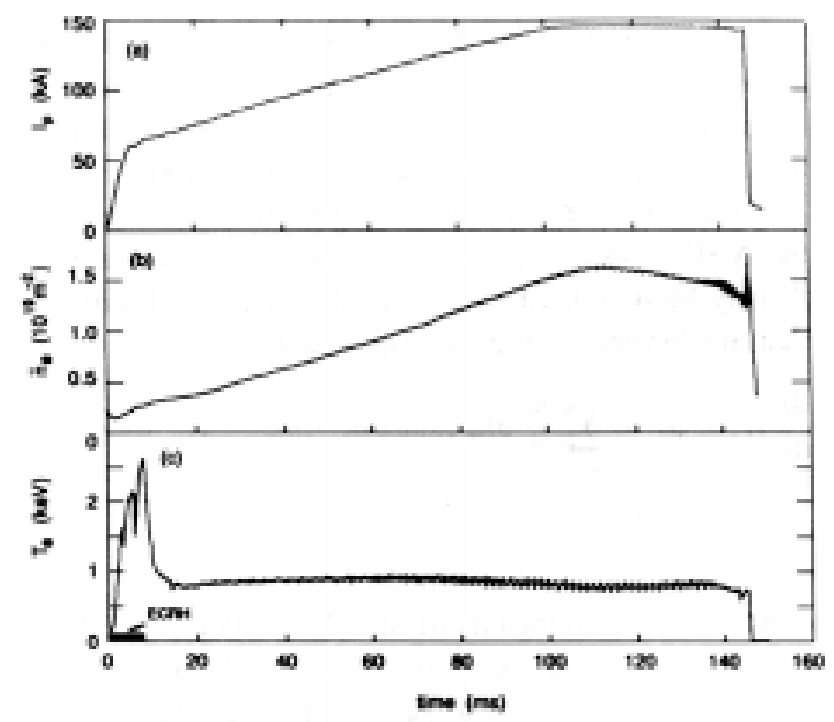

Fig. 3 a) Plasma current of discharge R19910423.057. A disruption occurs at $t=147 \mathrm{~ms}$.

b) Line integrated density from the central channel of the 19 -channel interferometer at $\mathrm{R}=0.72 \mathrm{~m}$. Sawteeth can be seen from $80 \mathrm{~ms}$ (before the flat-top of the plasma current is reached) to $137 \mathrm{~ms}$, when strong MHD activity sets in.

c) Central channel of the 20-channel ECE radiometer at $\mathrm{R}=0.735 \mathrm{~m}$. The initial peak in $\mathrm{T}_{\mathrm{e}}$ is due to ECR heating used to start up the plasma (from -2 to $8 \mathrm{~ms}$ ). The sawteeth are also visible here. 


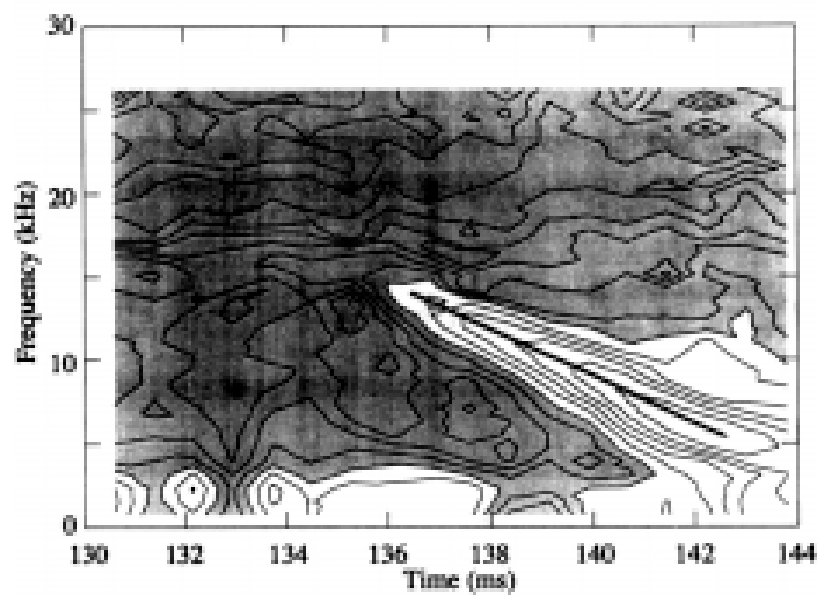

Fig. 4 Frequency versus time plot for ECE channel 7 at $\mathrm{R}=0.819 \mathrm{~m}$. The $\mathrm{m}=2$ mode is seen to decrease linearly in frequency from $15 \mathrm{kHz}$ at $135 \mathrm{~ms}$ to around $3 \mathrm{kHz}$ at $144 \mathrm{~ms}$, immediately preceding the disruption. 


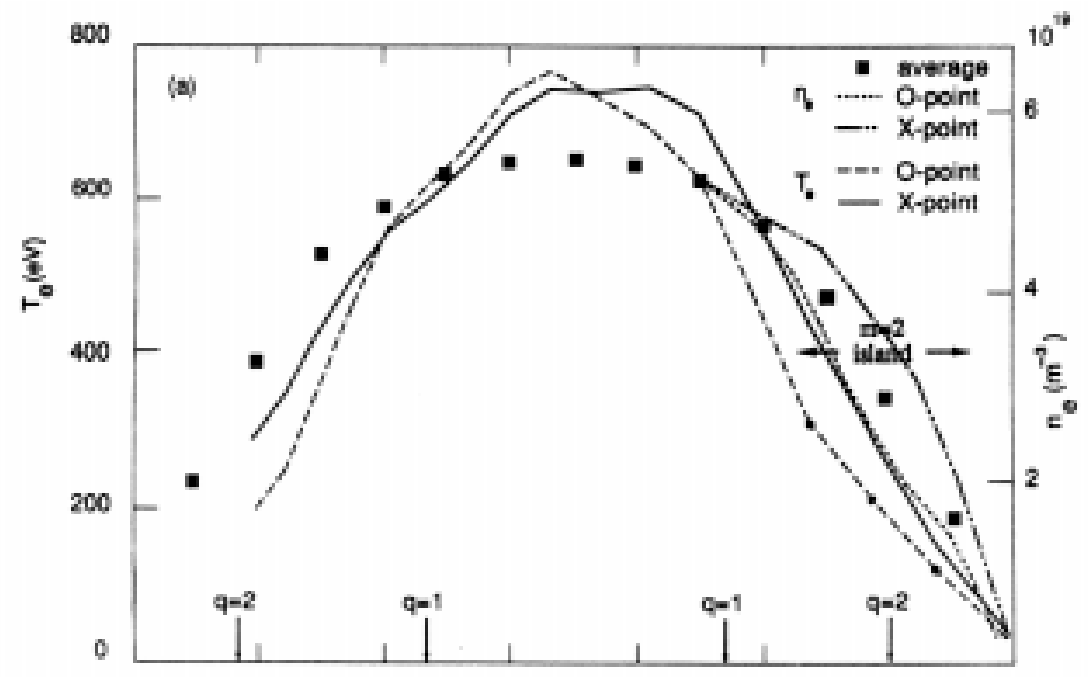

(b)

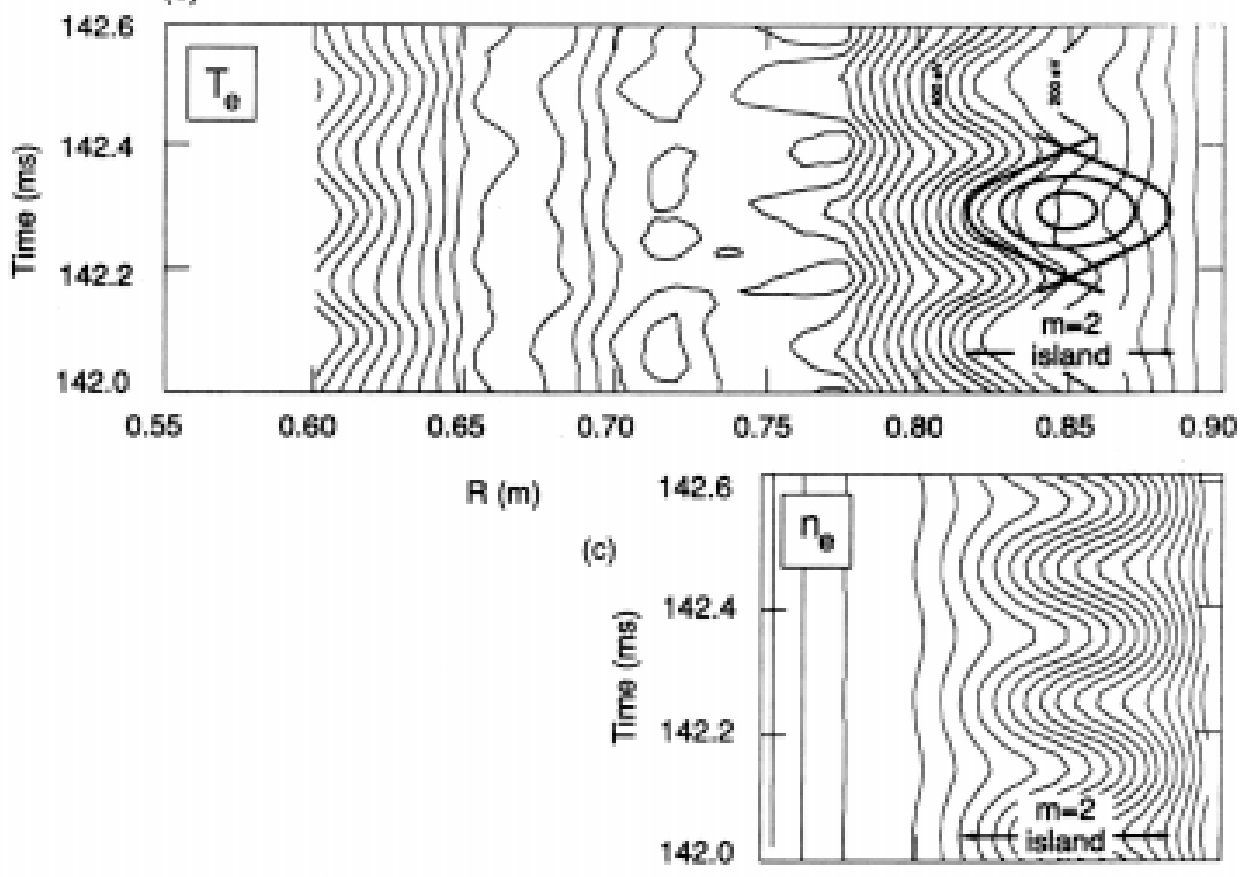

Fig. 5 a) $T_{e}$ profiles as measured with the ECE radiometer. The profiles correspond to two extrema in one oscillation period. The $\mathrm{q}=1$ and $\mathrm{q}=2$ radii are indicated, as well as the location of the $\mathrm{m}=2$ magnetic island as computed from the magnetic measurements. The $\mathrm{n}_{\mathrm{e}}$ profile at $\mathrm{t}=142 \mathrm{~ms}$ is reconstructed by Abel inversion.

b) Contour plots of $T_{e}$ and $n_{e}$ as a function of time and radius. The $n_{e}$ contours are constructed by superimposing the $\mathrm{n}_{\mathrm{e}}$ perturbation (see Fig. 6) on the profile in Fig. 5a. 

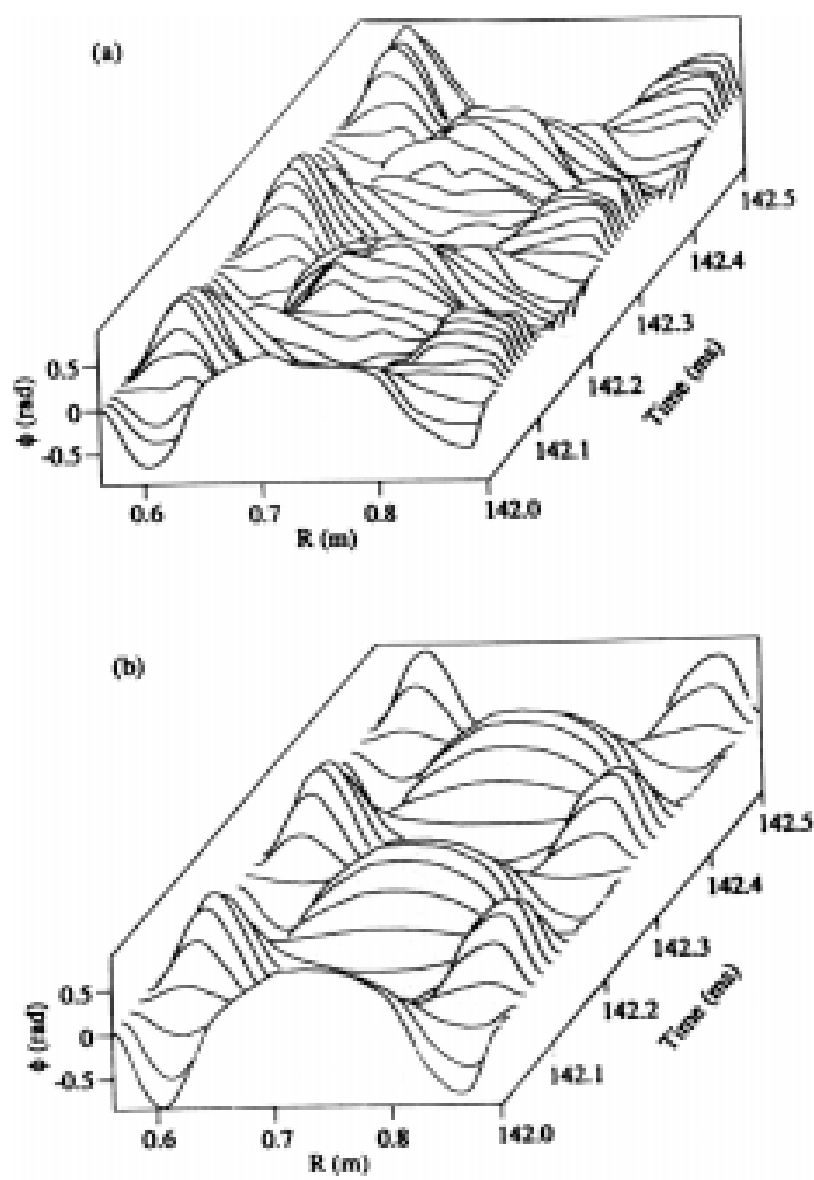

Fig. 6 a) The measured phase minus the average phase (averaging being done over the time window shown) of the interferometry data.

b) The reconstructed phase disturbance due to the presence of a rotating $\mathrm{m}=2$ island at $\mathrm{r}=13.5 \mathrm{~cm}$ (see text). The measured oscillations can be reasonably well explained by such an island.

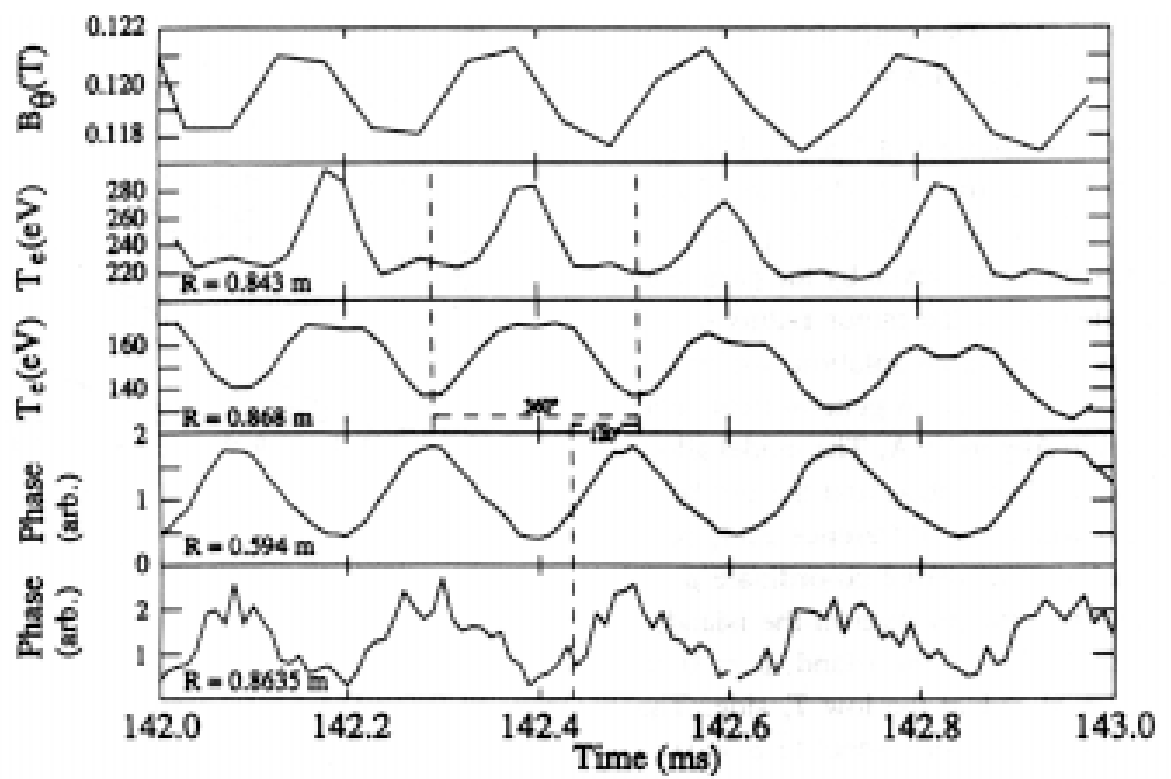

Fig. 7 Signals of $B_{\theta}(\theta=0), T_{e}(R=0.843 m), T_{e}(R=0.868 m), \bar{n}_{e}(R=0.594 m)$ and $\bar{n}_{e}(R=0.864 m)$. The $\bar{n}_{e}$ signals shown are raw data, they have arbitrary amplitude and offset, but the phase of the oscillations is correct as shown. The poloidal magnetic field and the temperature are measured at the same toroidal position, whereas the density is measured at a position separated $120^{\circ}$ toroidally. 


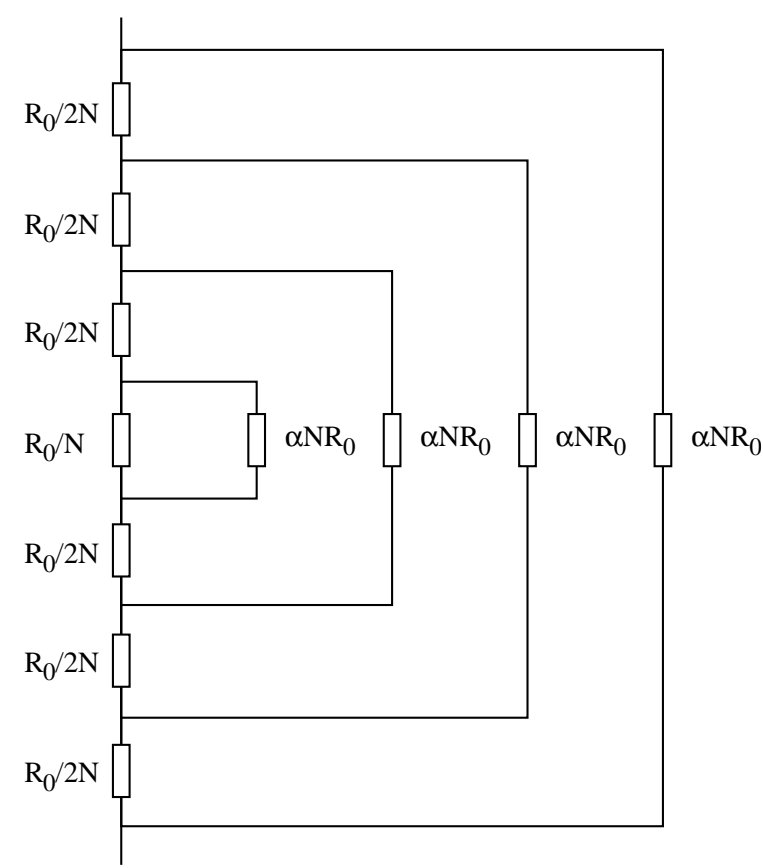

Fig. 8 A simple resistor network used to model the heat flux parallel and perpendicular to the field lines in the island. 

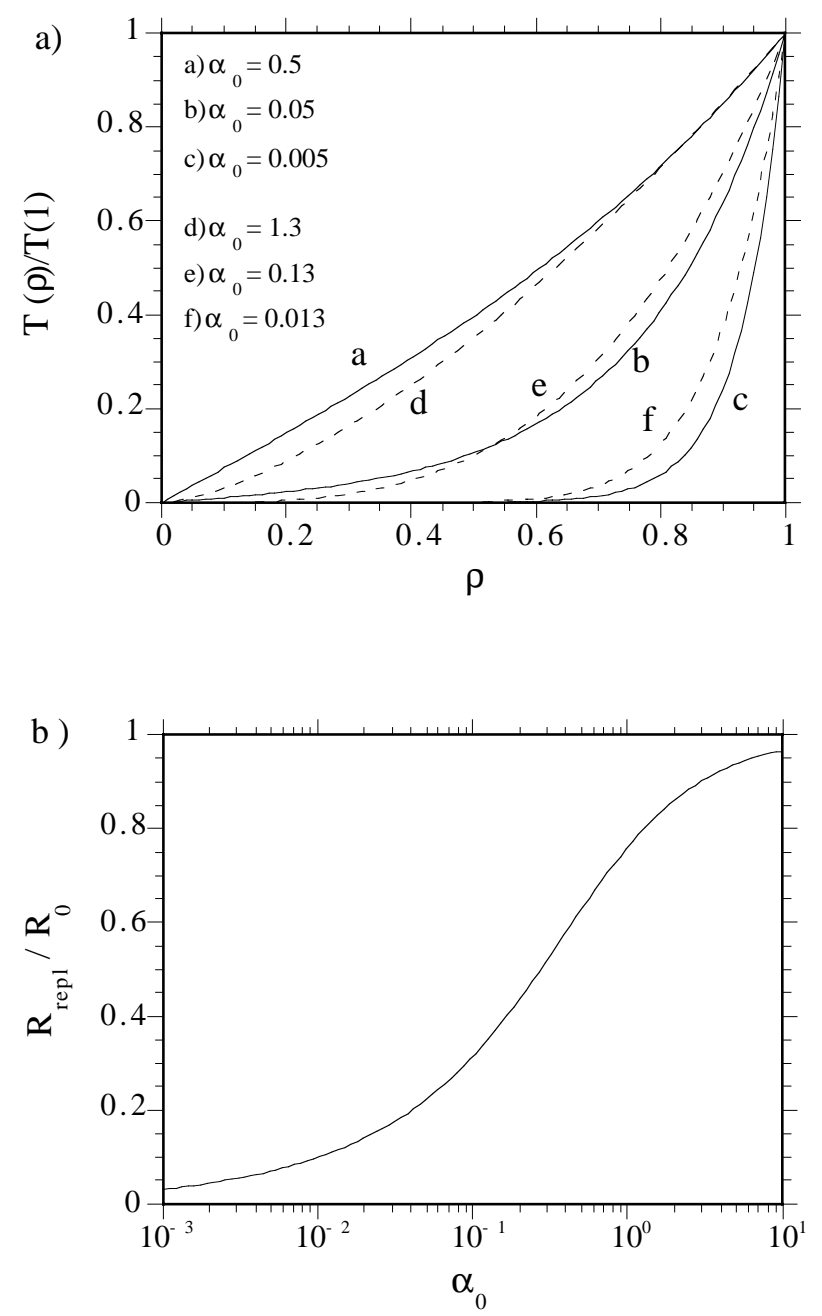

Fig. 9 a) The temperature profile across the island computed on the basis of the resistor model shown in Fig. 8 . T is normalized such that $\mathrm{T}(0)=0$ and $\mathrm{T}(1)=1$, and the T-profiles are antisymmetric with respect to $\rho=0$ : $\mathrm{T}(-\rho)=-\mathrm{T}(\rho)$. The full curves are for uniform $\alpha$, the dashed curves for a parabolic profile of $\alpha$. The curves are computed for various values of $\alpha_{0}$ as indicated. Clearly, significant flattening of $T(\rho)$ is only produced for $\alpha_{0}<0.48$ and 0.13 respectively.

b) The replacement resistance of the network $\left(R_{\text {repl }}\right)$ as a function of $\alpha_{0}$. 


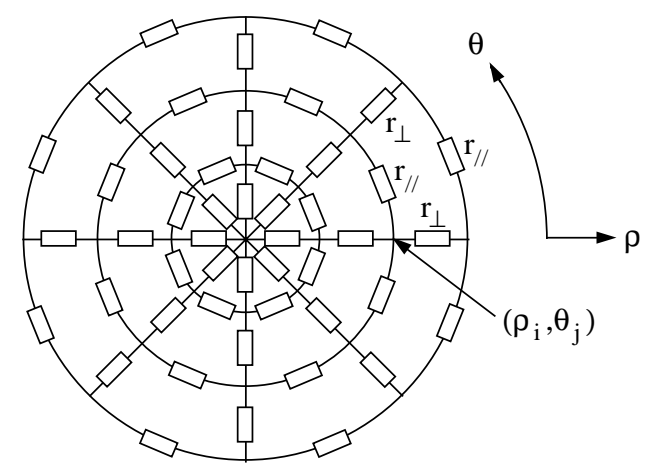

Fig. A.1 Network of resistors.

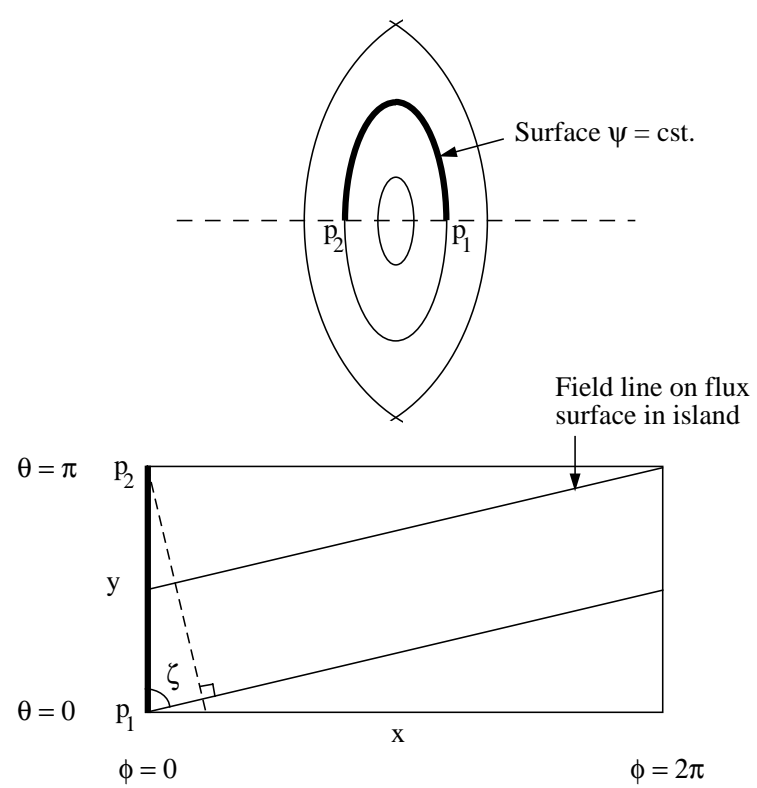

Fig. B.1 Flux surface geometry inside a magnetic island. 